\title{
HLA Class IA Histocompatibility Antigen Measurement
}

National Cancer Institute

\section{Source}

National Cancer Institute. HLA Class IA Histocompatibility Antigen Measurement. NCI

Thesaurus. Code C154746.

The determination of the amount of HLA class IA histocompatibility antigen present in a sample. 